\title{
CONGRUENCES WITH A COMMON MIDDLE ENVELOPE*
}

\section{BY MALCOLM FOSTER}

1. Introduction. Let $C$ and $\bar{C}$ be two rectilinear congruences whose corresponding rays $l$ and $\bar{l}$ are parallel; and let $M$ be the point on the unit sphere $S$ at which the normal is parallel to $l$ and $\bar{l}$. We refer the sphere to any isothermal system and take the linear element in the form $d s^{2}=e^{2 \lambda}\left(d u^{2}+d v^{2}\right)$. $\dagger$ Relative to the moving trihedral at $M$, whose $x$ axis is chosen tangent to the curve $v=$ const., the coordinates of the points in which $l$ and $\bar{l}$ pierce the $x y$ plane will be denoted by $(a, b)$ and $(\bar{a}, \bar{b})$, respectively. Distances on $l$ and $\bar{l}$ will be measured from these points, and the positive direction will be that which corresponds to the outward-drawn normal at $M$.

It is the purpose of this note to consider such pairs of congruences as $C$ and $\bar{C}$ when they have a common middle envelope, that is, when the distances to the middle points on $l$ and $\bar{l}$ are equal.

2. Condition that $C$ and $\bar{C}$ have a Common Middle Envelope. A necessary and sufficient condition that $C$ and $\bar{C}$ have a common middle envelope is that $\ddagger$

$$
\frac{\frac{\partial a}{\partial u}+\frac{\partial b}{\partial v}+a r_{1}-b r+2 \xi}{p_{1}}=\frac{\frac{\partial \bar{a}}{\partial u}+\frac{\partial \bar{b}}{\partial v}+\bar{a} r_{1}-\bar{b} r+2 \xi}{p_{1}} .
$$

This may be written

$$
\frac{\partial}{\partial u}(a-\bar{a})+\frac{\partial}{\partial v}(b-\bar{b})+(a-\bar{a}) \frac{\partial \lambda}{\partial u}+(b-\bar{b}) \frac{\partial \lambda}{\partial v}=0,
$$

which, upon multiplication by $e^{\lambda}$, becomes

$$
\frac{\partial}{\partial u}\left[e^{\lambda}(a-\bar{a})\right]=-\frac{\partial}{\partial v}\left[e^{\lambda}(b-\bar{b})\right] ;
$$

* Presented to the Society, February 23, 1935.

† Malcolm Foster, Rectilinear congruences referred to special surfaces, Annals of Mathematics, (2), vol. 25 (1923), pp. 159-180.

$\ddagger$ Foster, loc. cit., p. 163, equation (17). 
hence

$$
a-\bar{a}=e^{-\lambda} \frac{\partial R}{\partial v}, \quad b-\bar{b}=-e^{-\lambda} \frac{\partial R}{\partial u},
$$

where $R$ is an arbitrary function of $u$ and $v$. From (1) we have the following theorem.*

THEOREM 1. A necessary and sufficient condition that the congruences $C$ and $\bar{C}$ have a common middle envelope is that the congruence defined by the point $(a-\bar{a}, b-\bar{b})$ has for its middle envelope $a$ point, namely, the center of $S$.

3. Rotated Congruences. Let $C$ be the congruence defined by $(a, b)$; and let this point be rotated through an angle $\pi / 2$ about the corresponding normal to the point $(-b, a)+\dagger$ If $\bar{C}$ be the congruence defined by the point $(-b, a)$, we say $C$ and $\bar{C}$ constitute a pair of rotated congruences. We wish to determine those congruences $C(a, b)$, which with $\bar{C}(-b, a)$, have a common middle envelope. From (1) we must have

$$
a+b=e^{-\lambda} \frac{\partial R}{\partial v}, \quad b-a=-e^{-\lambda} \frac{\partial R}{\partial u} .
$$

The solution of these simultaneous equations will obviously give us the required condition:

$$
a=\frac{e^{-\lambda}}{2}\left(\frac{\partial R}{\partial v}+\frac{\partial R}{\partial u}\right), \quad b=\frac{e^{-\lambda}}{2}\left(\frac{\partial R}{\partial v}-\frac{\partial R}{\partial u}\right) .
$$

We therefore have the following result.

TheOREM 2. A necessary and sufficient condition that a congruence $C(a, b)$ and its rotated congruence $\bar{C}$ have a common middle envelope is that $a$ and $b$ have the values given in (2).

Suppose now that $C(a, b)$ has for its middle envelope the center of $S$. Then $a=e^{-\lambda}(\partial R / \partial v), b=-e^{-\lambda}(\partial R / \partial u)$. If $C(a, b)$ be rotated to $\bar{C}(-b, a)$, we know that $\bar{C}$ is a normal congruence. $\ddagger$

* Foster, loc. cit., p. 173.

$\dagger$ The direction of rotation is immaterial.

$\ddagger$ Foster, loc. cit., p. 166, Theorem 1. 
Let us now consider the middle point of the line joining $(a, b)$ and $(-b, a)$; its coordinates are $[(a-b) / 2,(a+b) / 2]$, or

$$
\left[\frac{e^{-\lambda}}{2}\left(\frac{\partial R}{\partial v}+\frac{\partial R}{\partial u}\right), \frac{e^{-\lambda}}{2}\left(\frac{\partial R}{\partial v}-\frac{\partial R}{\partial u}\right)\right] .
$$

Since (3) is identical with (2), we have the following theorem.

Theorem 3. Given a square $A B C D$, central with $M$, which lies in the $x y$ plane of the trihedral. If the point $A$ defines a congruence whose middle envelope is the center of $S$, so also does $C$, the opposite vertex, while the opposite vertices $B$ and $D$ define normal congruences; and the four points which bisect the sides of the square define four congruences with a common middle envelope.

4. $C$ and $\bar{C}$ Each Normal. Let $C$ and $\bar{C}$ be normal congruences. Then*

(4) $a=e^{-\lambda} \frac{\partial P}{\partial u}, \quad \bar{a}=e^{-\lambda} \frac{\partial \bar{P}}{\partial u}, \quad b=e^{-\lambda} \frac{\partial P}{\partial v}, \quad \bar{b}=e^{-\lambda} \frac{\partial \bar{P}}{\partial v}$.

By (1) and (4), a necessary and sufficient condition that the congruences $C$ and $\bar{C}$ have a common middle envelope is that

$$
\begin{aligned}
& a-\bar{a}=e^{-\lambda}\left(\frac{\partial P}{\partial u}-\frac{\partial \bar{P}}{\partial u}\right)=e^{-\lambda} \frac{\partial R}{\partial v}, \\
& b-\bar{b}=e^{-\lambda}\left(\frac{\partial P}{\partial v}-\frac{\partial \bar{P}}{\partial v}\right)=-e^{-\lambda} \frac{\partial R}{\partial u} .
\end{aligned}
$$

From (5), we have, from $\partial^{2} R / \partial u \partial v=\partial^{2} R / \partial v \partial u$, which is the condition of integrability,

$$
\frac{\partial^{2}}{\partial u^{2}}(P-\bar{P})+\frac{\partial^{2}}{\partial v^{2}}(P-\bar{P})=0 .
$$

We have therefore the following theorem.

THEOREM 4. A necessary and sufficient condition that the normal congruences (4) have a common middle envelope is that $(P-\bar{P})$ be a solution of Laplace's equation.

WESLEXAN UNIVERSITY

* Foster, loc. cit., p. 173. 